\title{
Analysis of energy transfer and ternary non-covalent filler/matrix/UV stabilizer interactions in carbon nanofiber and oxidized carbon nanofiber filled poly(methyl methacrylate) composites
}

\author{
I. Sedat Gunes ${ }^{\mathrm{a}, 1,2}$, César Pérez-Bolívar ${ }^{\mathrm{b}, 1}$, Guillermo A. Jimenez ${ }^{\mathrm{a}, 3}$, Omur Celikbicak $^{\mathrm{c}, 4}$, Fengyu Li ${ }^{\mathrm{b}}$, \\ Pavel Anzenbacher Jr. ${ }^{b}$, Chrys Wesdemiotis ${ }^{c}$, Sadhan C. Jana ${ }^{a, *}$ \\ a Department of Polymer Engineering, The University of Akron, Akron, OH 44325, USA \\ ${ }^{\mathrm{b}}$ Department of Chemistry and Center for Photochemical Sciences, Bowling Green State University, Bowling Green, OH 43403, USA \\ ${ }^{\mathrm{c}}$ Department of Chemistry, The University of Akron, Akron, $\mathrm{OH} 44325$, USA
}

\section{A R T I C L E I N F O}

\section{Article history:}

Received 8 August 2011

Received in revised form 7 September 2011

Accepted 10 September 2011

Available online 17 September 2011

\section{Keywords:}

Nanocomposites

Noncovalent interactions

Carbon nanofiber

\begin{abstract}
A B S T R A C T
Ternary non-covalent interactions between carbon nanofibers (CNFs), oxidized carbon nanofibers (oxCNFs), poly(methyl methacrylate) (PMMA) chains, and benzotriazole-containing UV stabilizers were analyzed using Fourier-transform infra red spectroscopy (FTIR), time-resolved fluorescence emission spectroscopy, and fluorescence lifetime imaging microscopy. The results indicated that PMMA chains form hydrogen bonds both with ox-CNF fibers and the UV stabilizer molecules. It was also determined that UV stabilizers strongly interact with CNF particles via $\pi-\pi$ interactions. The extent of $\pi-\pi$ and hydrogen bonding interactions was determined to be lower between ox-CNF particles and UV stabilizers due to less perfect graphitic structure of the former. The morphology of the composites indicated that the hydrogen bonds between PMMA chains and ox-CNF particles resulted in highly improved state of filler dispersion in ox-CNF/PMMA composites.
\end{abstract}

(c) 2011 Elsevier Ltd. All rights reserved.

\section{Introduction}

Poly(methyl methacrylate) (PMMA) composites filled with carbonaceous nano-size fillers have been attracting growing attention due to their potential use in construction of flexible solar cell electrodes [1]. The PMMA composites used in the solar energy applications are multi-component systems containing stabilizers and additives in the PMMA matrix. Among the additives, ultraviolet (UV) stabilizers play the most dominant role in the final composition and ensure that the resulting composites have the required long-term UV stability, preferably on the order of decades. Thus, non-covalent and photophysical processes associated with the UV stabilizers and the PMMA chains with the nano-size fillers

\footnotetext{
* Corresponding author. Tel.: +1 330972 8293; fax: +1 3302582339.

E-mail address: janas@uakron.edu (S.C. Jana).

1 These authors equally contributed to the presented work

2 Present address: 3M Corporate Research Process Laboratory, 3M Center, St. Paul, MN 55144, USA.

3 Present address: Laboratory of Polymers (POLIUNA), School of Chemistry, Universidad Nacional, Heredia, 86-3000 Costa Rica.

${ }^{4}$ Permanent address: Department of Chemistry, Hacettepe University, Beytepe, Ankara 06800, Turkey.
}

are fundamentally important for the properties and performance of the PMMA composites for optoelectronic applications. In addition, understanding of non-covalent interactions and photophysical stability of these composites could shed light on mechanisms of light-induced energy transfer in other polymer nanocomposites $[2,3]$. Note also that favorable interactions between polymer chains and nano-size filler particles are essential for the preparation of nanocomposites with highly dispersed filler particles [4-9]. Our earlier studies $[10,11]$ suggest that favorable filler-matrix interactions between oxidized carbon nanofiber (ox-CNF) particles and poly(methyl methacrylate) (PMMA) improved the degree of filler dispersion, which in turn improved the engineering properties of the composites.

In this work, we analyzed non-covalent interactions and lightinduced energy transfer between PMMA, UV stabilizer additives, and carbon nanofibers. Fourier-transform infra red spectroscopy (FTIR) and fluorescence emission spectroscopy methods were utilized to analyze the interactions between the components. Our earlier work [12] indicated that fluorescence emission spectroscopy was a powerful method to characterize polymer nanocomposites due to its high sensitivity and high temporal resolution. Since PMMA itself does not display fluorescence emission, it is an ideal polymer matrix to analyze interactions of UV stabilizers via 
fluorescence spectroscopy. However, due to the lack of emission from the PMMA it is not possible to study the nature of the interactions between the filler particles and the PMMA chains. Here we used FTIR analysis to identify the nature of interactions between the fillers and PMMA. The combination of different spectroscopic techniques allowed for complementary and broader understanding of the interactions taking place between the PMMA and the components of the composites.

The rationale for focusing on the interactions of carbon nanofibers with UV stabilizers is presented as follows. Many commercial resins are sold with UV stabilizers and other organic additives present in them. However, a majority of research reports on nanoparticle dispersion in commercial polymers pay close attention to the state of dispersion of nanofillers and draw inferences primarily from the physical and chemical interactions between the polymer chains and the nanoparticles. The role of potential interactions of organic additives, such as UV stabilizers, with nanofillers is often ignored. Such interactions with organic additives may limit the scope of polymer-filler interactions. In view of this, the present paper placed a primary focus on the analysis of interactions between carbon nanofibers and the UV stabilizer in PMMA and not so much on particle dispersion. Detailed prior work exists on analysis of particles dispersion in several polymer systems [4-13].

\section{Experimental}

\subsection{Materials}

PMMA (Perspex CP-80) pellets were kindly donated by Lucite International (Cordova, TN) with melt flow index of 2.2 (ASTM D 792 , at $230{ }^{\circ} \mathrm{C}$ and under the load of $3.8 \mathrm{~kg}$ ) and density of $1.19 \mathrm{~g} /$ $\mathrm{cm}^{3}$. The weight average molecular weight of PMMA determined by gel permeation chromatography was found to be $144,700 \mathrm{~g} / \mathrm{g}$ mol [10]. Vapor grown carbon nanofiber (CNF, Pyrograph III ${ }^{\circledR}$ PR-24-PS) and oxidized carbon nanofiber (ox-CNF, Pyrograph III ${ }^{\circledR}$ PR-24-PSox) were obtained from Applied Sciences, Inc. (Cedarville, $\mathrm{OH}$ ) with diameters of $60-200 \mathrm{~nm}$ and lengths of $30-100 \mu \mathrm{m}$. The $\mathrm{x}$-ray photoelectron spectroscopy (XPS) data [11] indicated that the surfaces of CNF particles were almost entirely composed of graphitic carbon. Oxygen-rich polar functional groups, such as $\mathrm{C}-\mathrm{O}-\mathrm{C}, \mathrm{C}=\mathrm{O},-\mathrm{COOR}$, and $-\mathrm{COOH}$, were determined on the surface of ox-CNF particles. The XPS data [11] indicated that the molar ratio of carbon atoms to oxygen atoms on the surfaces of CNF and ox-CNF were 66.7 and 7.7 respectively. Controlled nanofiber oxidation also eliminated a significant portion of amorphous carbon potentially present on the fibers [11], which in turn produced a relatively less entangled morphology in as-received oxCNFs. Note also that oxidation of carbon nanofibers partially broke down the continuity of the graphitic structure of CNFs and resulted in lower intrinsic electrical conductivity of ox-CNF fibers [11].

\subsection{Sample preparation}

Previous studies established the advantages of low shear chaotic mixers for preparation of polymer nanocomposites comprising fibrous nanofillers [11,14]. It was observed that low shear rate ranging from $4.8 \mathrm{~s}^{-1}$ at the rotor wall to $2.7 \mathrm{~s}^{-1}$ at the mixing chamber wall preserved the nanofiber length. In addition, the chaotic trajectory of the fluid improved both dispersive and distributive mixing [15]. Consequently, the composites produced in chaotic mixer offered better mechanical, thermal and electrical conductivity properties compared to materials prepared in commercial internal mixers under similar shear rate [11]. Thus composites of $\mathrm{CNF}$ and ox-CNF were prepared using chaotic mixer.
Detailed information on mixing protocol and the effects of mixing time on product properties were reported $[11,14]$.

\subsection{Characterization}

The scanning electron microscopy (SEM) images were acquired with SEM S-2150 microscope (Hitachi, Japan) at $20 \mathrm{kV}$ after sputter coating the cryogenically fractured specimens with silver using a K575x sputter coater (Emitech, UK) under argon atmosphere. Thin slices ( $\sim 50-70 \mu \mathrm{m}$ thick) of specimens were analyzed using a Leitz Laborlux 12 Pol S optical microscope (OM) (Oberkochen, Germany) in transmission mode, and the images were recorded with a digital camera (Diagnostic Instruments, Sterling Heights, MI). The FTIR spectra were recorded using Nicolet 4700 FTIR Spectrometer with TGS Detector (Thermo Scientific, Waltham, MA), and in micro-attenuated total reflectance (microATR) mode. The data were recoded using a MicroATR fixture (SensIR Technologies, DuraScope, Danbury, CT) with a diamond crystal. Scans were acquired at a resolution of $4 \mathrm{~cm}^{-1}$ and ATR correction was applied. Fluorescence emission spectra were recorded on a single-photoncounting spectrofluorimeter (FL/FS 900, Edinburgh Analytical Instruments, Livingston, UK) equipped with a CW $450 \mathrm{~W}$ xenon arc
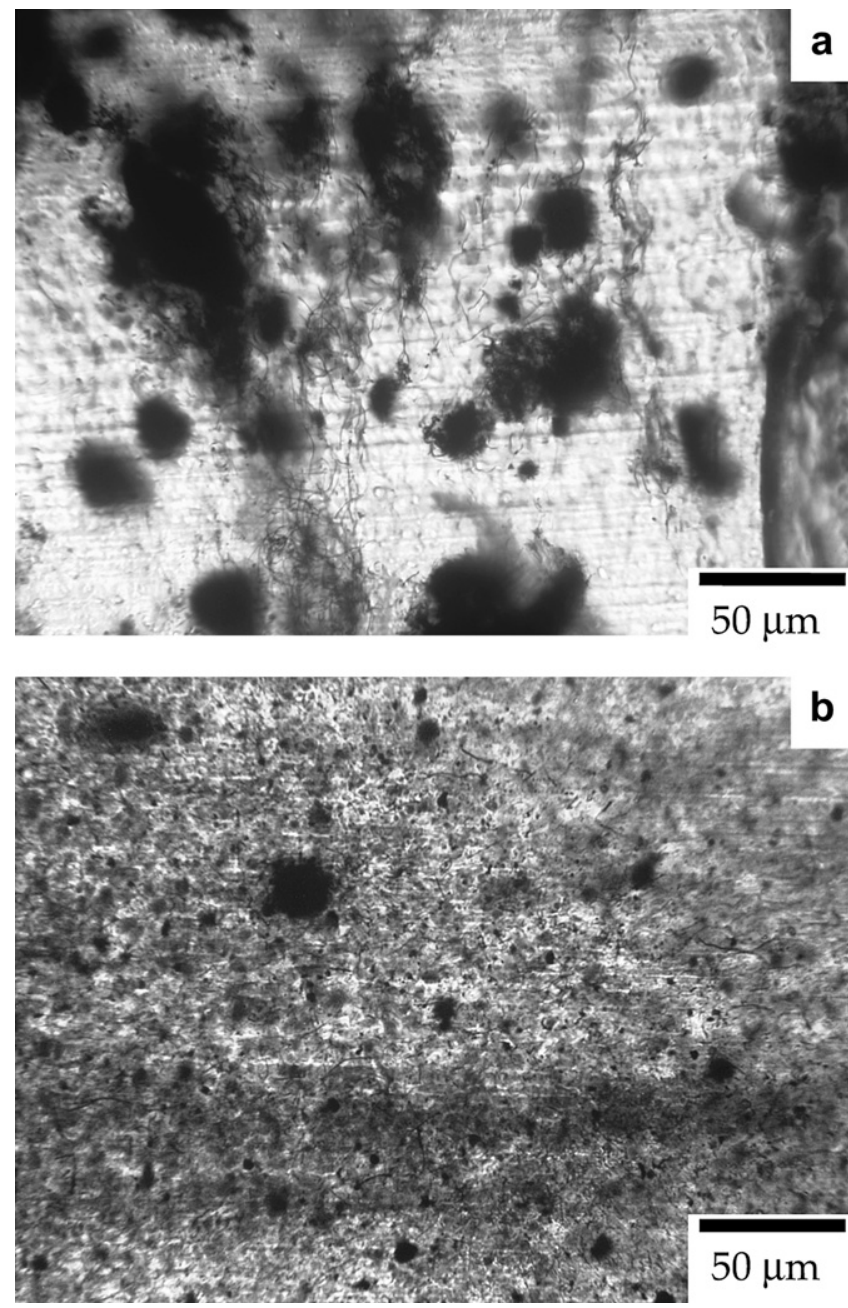

Fig. 1. Optical microscope images of PMMA composites with 1 wt\% carbon nanofiber The images were taken at low magnification in order to evaluate the overall dispersion of the filler particles. (a) CNF/PMMA (b) ox-CNF/PMMA. Note the much improved dispersion of filler particles in the case of ox-CNF/PMMA composites. 
lamp. The focal length for both excitation and emission monochromators was $300 \mathrm{~mm}$. Both monochromators had a CzernyTurner design triple grating turret. The resolution for the monochromators was $0.05 \mathrm{~nm}$. The detector was a single photon counting photomultiplier. The steady-state lifetime measurements were recorded in a time-correlated single photon counting spectrofluorimeter from Edinburgh Analytical Instruments (FL/FS 900) with a photomultiplier tube in a Peltier-cooled housing, the sources used were measured a $375 \mathrm{~nm}$ laser diode (PicoQuant, LDH-D-C375B) and a nanosecond Hydrogen pulse lamp from Edinburgh Instruments. The lifetime measurements were repeated ten times. Their arithmetic average and the standard deviation were calculated. The assignment of peak locations and corresponding fitting of fluorescence spectra were performed with a curve fitting and data analysis software (Fityk 0.7.7). A Waters SYNAPT high definition
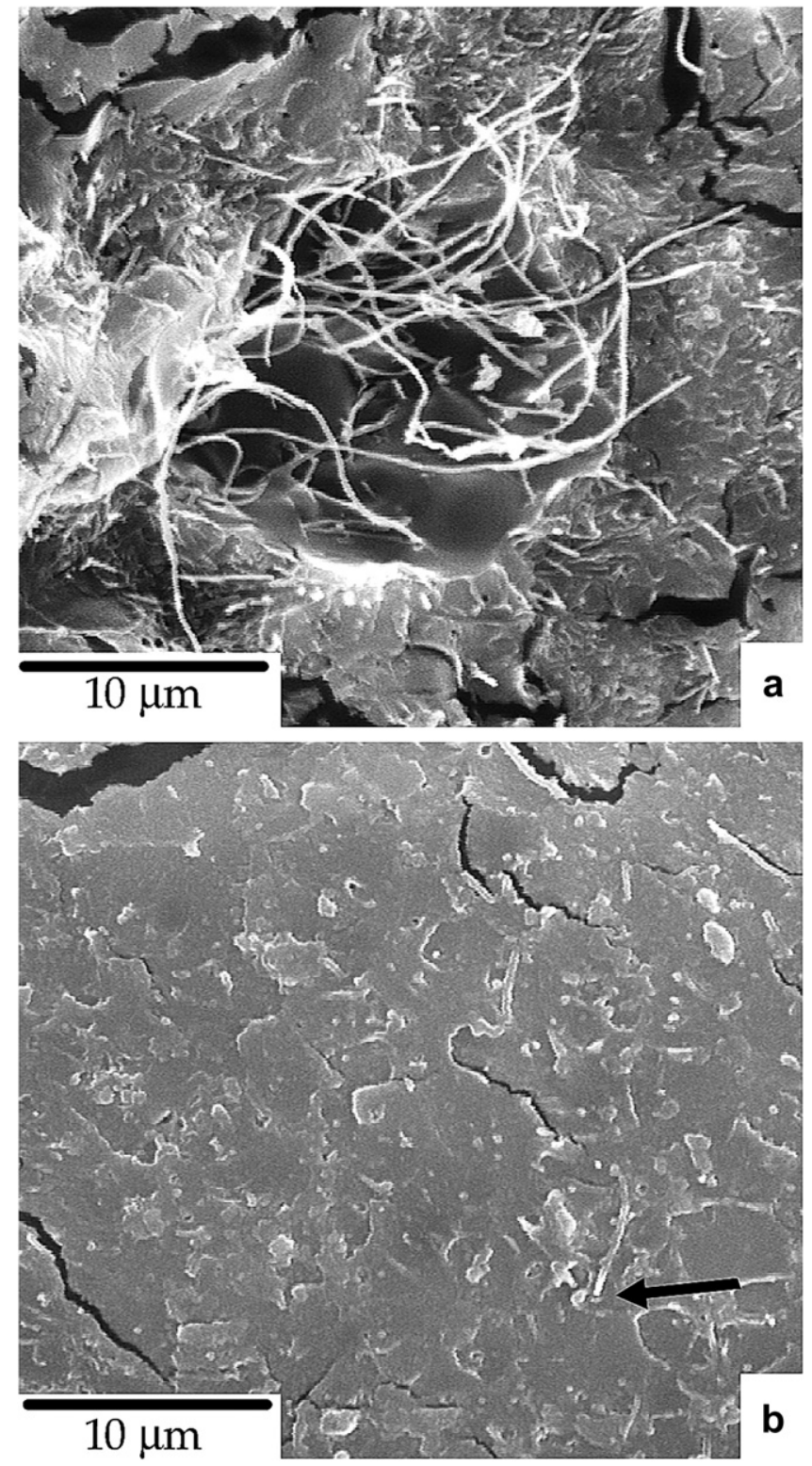

Fig. 2. SEM images of cryogenically fractured surfaces of PMMA composites with 4 wt $\%$ carbon nanofiber. (a) CNF/PMMA (b) ox-CNF/PMMA. The presence of a number of aggregates of isolated fibers is clearly observed in (a), whereas only the tips of the fibers are seen in the case of ox-CNF/PMMA composites, as identified by the arrow in (b).
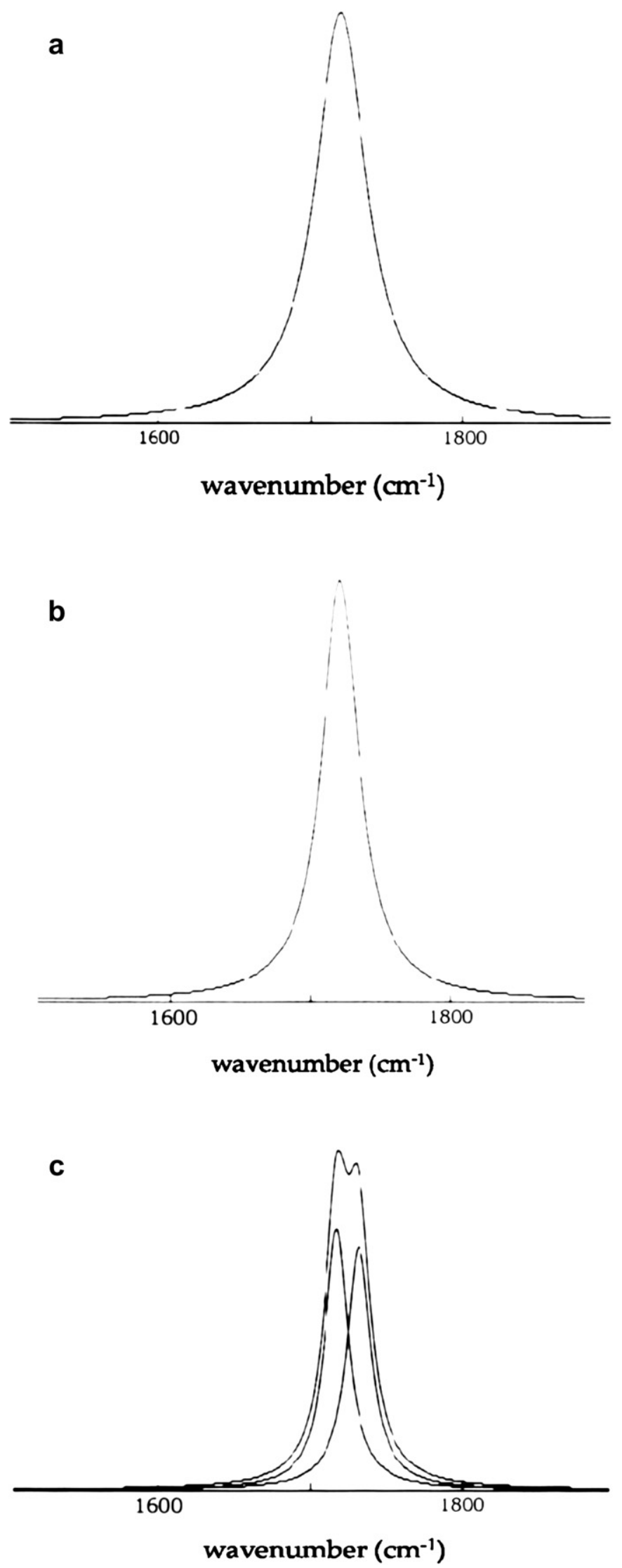

Fig. 3. Carbonyl stretching regions of FTIR spectra of a) pristine PMMA b) CNF/PMMA composite with $1 \mathrm{wt} \%$ filler content c) ox-CNF/PMMA composite with $1 \mathrm{wt} \%$ filler content. The presence of a single peak located at around $1720 \mathrm{~cm}^{-1}$ was observed in pristine PMMA and CNF/PMMA composites. The formation of a second peak located at around $1731 \mathrm{~cm}^{-1}$ was observed in ox-CNF/PMMA which can be attributed to the presence of carbonyl groups which were hydrogen bonded to carboxyl groups on ox-CNF. 
mass spectrometer (HDMS; Waters Corp., Manchester, UK) equipped with an ESI source was used for the quadrupole time-of-flight (Q-ToF) mass spectrometry experiments. The PMMA sample was dissolved in chloroform to a concentration of $2 \mathrm{mg} / \mathrm{mL}$ and then diluted to $1 \mathrm{mg} / \mathrm{mL}$ using methanol. The final solution was introduced directly to the ESI source using a syringe pump at a flow rate of $20 \mu \mathrm{L} / \mathrm{min}$. Mass spectra were acquired in positive ion mode. The capillary voltage was $3.5 \mathrm{kV}$, and the ion source block and nitrogen desolvation gas temperatures were set to $120{ }^{\circ} \mathrm{C}$ and $150{ }^{\circ} \mathrm{C}$, respectively. The elemental analysis was performed with a Perkin Elmer 2400 Series II CHNS/O system (Waltham, MA), equipped with a Perkin Elmer EA Data Manager software. The Fluorescence lifetime micrographs were recorded on a PicoQuant LSM Upgrade Kit (MicroTime 200/PDL-800-B/PicoHarp 300) for a FluoView 1000 Laser Scanning Microscope (Olympus), both controlled by SymPhoTime 5.13. The excitation source was a $375 \mathrm{~nm}$ laser diode (PicoQuant, LDH-D-C-375B) at $20 \mathrm{MHz}$ repetition pulses and the emission was recorded through a $400 \mathrm{~nm}$ pass filter. The image recorded was based on the fluorescence lifetime distribution of the components in the illuminated area. The bright field images were measured using laser excitation at $458 \mathrm{~nm}, 515 \mathrm{~nm}$, and $633 \mathrm{~nm}$.

\section{Results and discussion}

\subsection{State of filler dispersion and morphology of fractured surfaces}

The state of filler dispersion in PMMA composites and the morphology of fractured surfaces suggested the presence of favorable filler-matrix interactions in composites of ox-CNF, as discussed previously [11]. A set of representative optical microscope images of PMMA composites is presented in Fig. 1 showing the significantly better state of dispersion of ox-CNF in PMMA. Similar observations were made in the case of composites of shape memory polyurethanes as well [16]. The fractured surfaces of CNF/ PMMA specimens revealed that the aggregates of CNF were not fully wetted by the polymer (Fig. 2a). This provided indirect evidence of poor adhesion between CNF and PMMA which in turn resulted in pull-out of fibers during fracture. On the other hand, all ox-CNF fibers appeared wetted in ox-CNF/PMMA composites (Fig. 2b). We previously observed similar differences between the morphologies of fractured surfaces of composites of CNF/SMPU and ox-CNF/SMPU [16].
Table 1

Elemental analysis of PMMA. C: carbon, H: hydrogen, O: oxygen, $\mathrm{N}$ : nitrogen. The values for PMMA sample were the arithmetic average of three measurements.

\begin{tabular}{|c|c|c|c|c|}
\hline & \multicolumn{4}{|c|}{ Elemental composition (wt \%) } \\
\hline & $\mathrm{C}$ & $\mathrm{H}$ & $\mathrm{O}$ & $\mathrm{N}$ \\
\hline PMMA sample (Perspex $\left.{ }^{\circledR} \mathrm{CP}-80\right)$ & 59.4 & 8.3 & 32.1 & 0.2 \\
\hline Pure PMMA (theoretical) & 59.98 & 8.06 & 31.96 & 0.0 \\
\hline
\end{tabular}

\subsection{Analysis of composites via FTIR}

The carbonyl groups in PMMA were thought to participate in dipole-dipole interactions [17]. In addition, the formation of hydrogen bonding between the carbonyl groups of PMMA and the carboxylic acid groups were previously observed [18]. In view of these findings, we focused on the carbonyl stretching regions of the FTIR spectra of PMMA and its composites with CNF and ox-CNF. The expanded views of carbonyl stretching bands of pristine PMMA and its composites with CNF and ox-CNF, each with $1 \mathrm{wt} \%$ filler content, are presented in Fig. 3a-c. Pristine PMMA and CNF/PMMA composites exhibited only a single carbonyl peak. On the other hand, a bifurcated carbonyl stretching region was observed in the case of ox-CNF/PMMA composites (Fig. 3c). In view of the previous studies on interactions between carbonyl and carboxylic acid groups [18-20], we infer from the data presented in Fig. $3 c$ that a fraction of carbonyl groups in PMMA, purportedly the ones in the proximity of surfaces of ox-CNF particles, formed hydrogen bonds with the carboxylic acid groups of ox-CNF. These hydrogen bonds are thought to have promoted dispersion of ox-CNF particles in PMMA as inferred from Fig. 1b and ramified into diffused fillerpolymer interfaces as evidence of stronger filler-polymer interactions (Fig. 2b).

\subsection{Analysis of composites via fluorescence emission spectroscopy}

The type of and the content of UV stabilizers in PMMA samples were determined by mass spectroscopy and elemental analysis [21]. Note that the most common UV stabilizers in commercial PMMA samples were benzotriazole containing additives [22]. Mass spectrometry of PMMA clearly indicated the presence of impurities and additives (Fig. 4). The proposed chemical formula of the commercial additive in PMMA was acquired from the accurate mass measurement of the ion at the mass-to-charge ratio $(\mathrm{m} / \mathrm{z})=323$



Fig. 4. Positive ion mode ESI-Q-ToF spectrum of PMMA sample. Note that the molecular weight of the repeating unit of PMMA is 100. 


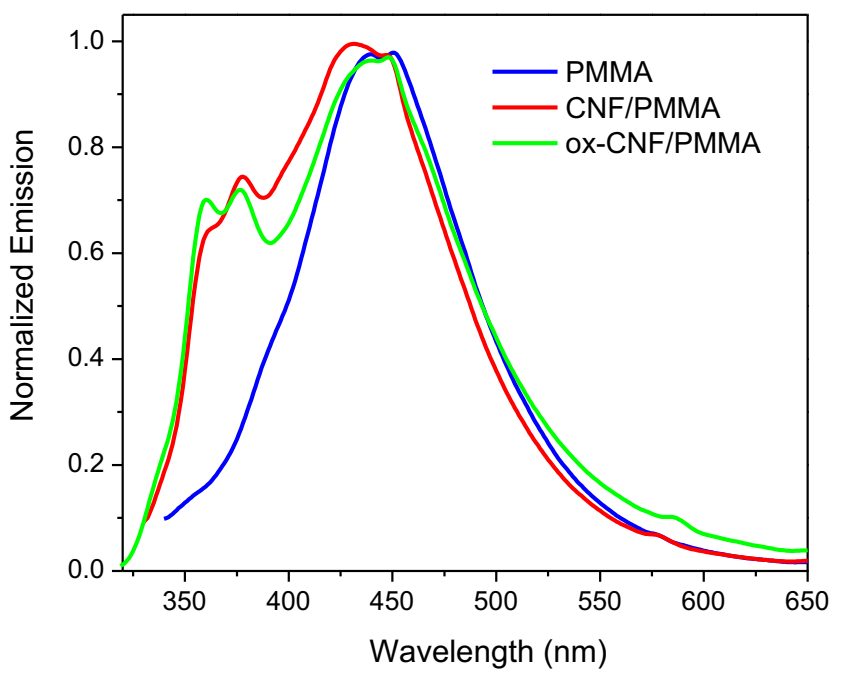

Fig. 5. Emission spectra of PMMA, and the CNF/PMMA and ox-CNF/PMMA composites. The spectra were recorded at room-temperature using a Xenon lamp as a source. The excitation wavelength was $290 \mathrm{~nm}$.

from the PMMA sample (Fig. 4), and tandem mass spectrometry experiments (data not shown). The accurate mass measured was 323.202, which most closely matched the calculated mass of the radical ion $[\mathrm{M}]^{+}$of 2 -(2H-benzotriazole-2-yl)-4-(1, 1, 3, 3-tetramethylbutyl)-phenol $\left(\mathrm{C}_{20} \mathrm{H}_{25} \mathrm{~N}_{30}\right.$, calculated mass 323.200$)$. The measured mass and the suggested chemical formula closely coincided with a common UV stabilizer which is usually present in commercial PMMA grades in the form of Uvinul ${ }^{\circledR} 3029$, a product of BASF [23]. Abundant signals were also observed above $900 \mathrm{~m} / \mathrm{z}$ which were assigned to the lower molecular weight PMMA chains. The observed signals below $900 \mathrm{~m} / \mathrm{z}$ suggested the presence of other minor additives or impurities present in the PMMA sample.

In order to obtain further supporting evidence for the presence of benzotriazole containing additives in PMMA, we next referred to elemental analysis. Note that benzotriazole contains nitrogen while PMMA does not. The elemental analysis results presented in Table 1 supplies evidence for the presence of nitrogen in PMMA sample which is a further supportive evidence for the presence of benzotriazole containing additives. Note that the UV stabilizer contents in PMMA samples are usually on the order of $0.2 \mathrm{wt} \%$ [22] which also coincides with the relatively low nitrogen content.

The emission spectra of PMMA and the CNF/PMMA and ox-CNF/ PMMA composites are presented in Fig. 5. The samples were excited at $290 \mathrm{~nm}$ and the spectra clearly showed an extra shoulder in the emission in the composites in respect to the unfilled PMMA. The emission is thought to arise from the non-covalent, nonspecific interactions of UV stabilizers with the filler particles. The shifts on the emission maxima for the samples from $442 \mathrm{~nm}$ to $451 \mathrm{~nm}$ respectively for PMMA, to $431 \mathrm{~nm}$ and $448 \mathrm{~nm}$ in CNF/ PMMA, to the formation of just one maximum in ox-CNF/PMMA at $443 \mathrm{~nm}$ indicate that the degree of interactions between the stabilizers and the PMMA chains change due to the nature of the fillers. Broadening in the emission bands of the composites was also observed which indicated the presence of strong $\pi-\pi$ interactions which reduced the definition of the emission bands. These differences in the shape of the spectra were considered in selecting the different wavelengths for the emission lifetime analysis. The analysis was performed for the broad bands that peak over $420 \mathrm{~nm}$ for the samples. This region is the only one in common between the composites and the neat PMMA. The excitation wavelength for the analysis was $375 \mathrm{~nm}$ from a laser diode or a microsecond pulse Xenon lamp.

\subsection{Time-resolved fluorescence emission spectroscopy and fluorescence lifetime imaging microscopy}

The ternary non-covalent interactions and energy transfer between UV stabilizers, PMMA chains, filler particles were analyzed with the time-resolved fluorescence emission spectroscopy (TRES) (Table 2). The fluorescence decay experiments on pristine PMMA samples indicated two distinct lifetimes. The first lifetime is considerably shorter ( $\sim 1 \mathrm{~ns}$ ) as compared to the second one ( $\sim 6 \mathrm{~ns})$. Their relative contributions were observed to be about $45 \%$ and $55 \%$. Note that it was previously observed that two distinct forms of benzotriazole containing UV stabilizers were present in polar solvents - 'free' UV stabilizers which form intramolecular hydrogen bonds and 'bonded' UV stabilizers which form intermolecular hydrogen bonds with the polar solvent molecules [24]. In view of this, we attributed the origin of the short lifetime to the presence of 'free' UV additives which did not interact with the surrounding media and formed intramolecular hydrogen bonds. The longer lifetime was considered to be originating from the 'bonded' molecules which interacted with the surrounding PMMA chains by forming intermolecular hydrogen bonds. The longer lifetime observed in 'bonded' UV additives has been usually attributed to the formation of a more delocalized electronic state [25].

The CNF-filled PMMA composites also indicated the presence of two distinct lifetimes (Table 2). However, a comparison of the lifetimes determined in pristine PMMA and CNF/PMMA composites indicated that the presence of CNF particles appeared to have different extents of influence on the observed lifetimes. The magnitude of the shorter lifetime appeared to be relatively unaffected by the presence of CNF particles, although the second, longer lifetime became even longer in the presence of them. Note that the relative contributions of the two life-times were also significantly affected by the presence of CNF particles. The contribution of the

Table 2

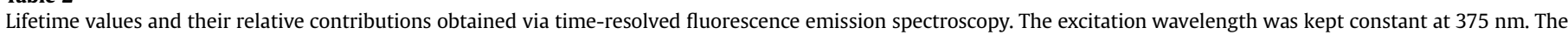

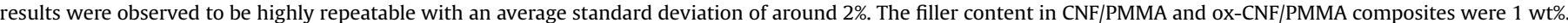

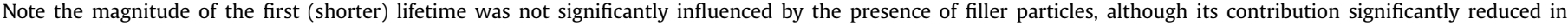
composites. The magnitude and the contribution of the second (longer) lifetime significantly increased in presence of CNF particles.

\begin{tabular}{|c|c|c|c|c|c|c|}
\hline \multirow[t]{2}{*}{ Emission Wavelength (nm) } & \multicolumn{2}{|l|}{ PMMA } & \multicolumn{2}{|l|}{ CNF/PMMA } & \multicolumn{2}{|l|}{ ox-CNF/PMMA } \\
\hline & Lifetime (ns) & Relative Contribution (\%) & Lifetime (ns) & Relative Contribution (\%) & Lifetime (ns) & Relative Contribution (\%) \\
\hline \multirow[t]{2}{*}{410} & 0.94 & 44 & 0.90 & 18 & 1.09 & 29 \\
\hline & 5.81 & 56 & 8.21 & 82 & 6.76 & 71 \\
\hline \multirow[t]{2}{*}{430} & 0.99 & 43 & 1.04 & 18 & 1.11 & 27 \\
\hline & 5.92 & 57 & 8.90 & 82 & 5.62 & 73 \\
\hline \multirow[t]{2}{*}{450} & 1.04 & 40 & 1.24 & 23 & 1.28 & 28 \\
\hline & 6.20 & 60 & 8.87 & 77 & 6.01 & 72 \\
\hline
\end{tabular}


shorter lifetime was considerably reduced in the presence of CNF particles. The results indicated that a greater portion of the UV stabilizers appeared to be in 'bonded' state in CNF/PMMA composites which could be attributed to the $\pi-\pi$ interactions between CNF particles and the UV additives [26] in addition to hydrogen bonding between UV additives and PMMA chains.

The results on ox-CNF/PMMA composites (Table 2) also indicated that the UV stabilizers interacted with ox-CNF particles as
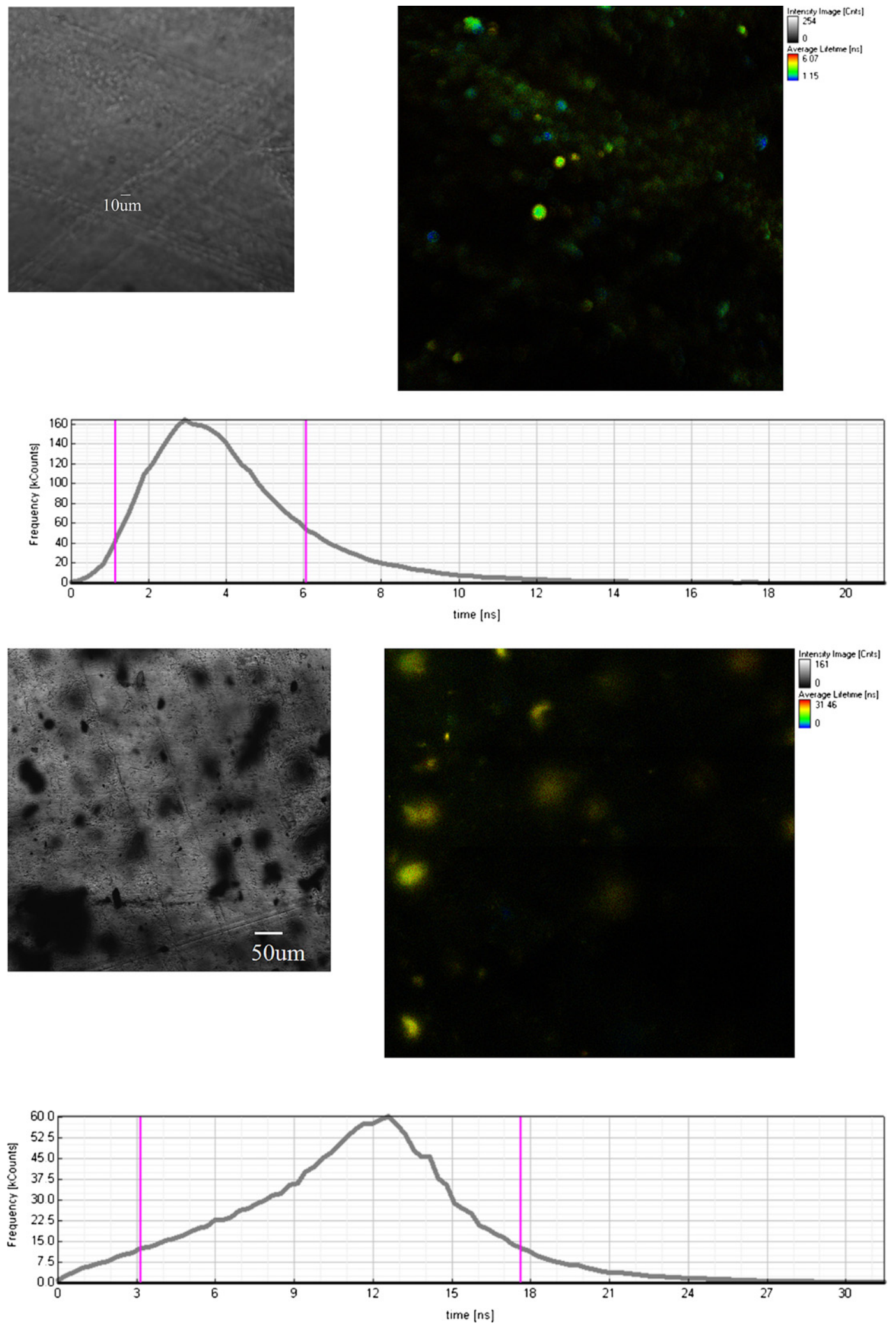

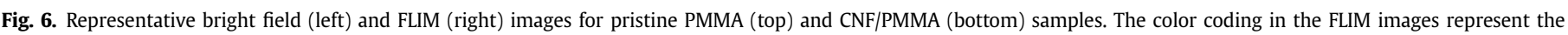
average lifetimes recorded for the emissive species excited at $375 \mathrm{~nm}$. 
well as with PMMA chains. The comparison of lifetimes and their relative contributions in pristine PMMA, CNF/PMMA, and ox-CNF/ PMMA composites revealed further insight on the nature and extent of non-covalent interactions. In view of the magnitude and relative contributions of the observed life-times, one might infer that the extent of the interactions between UV stabilizers and oxCNF particles were reduced as compared to those between UV stabilizers and CNF particles (Table 2). This, in turn, indicated that the dominant non-covalent interaction between the UV stabilizers and CNF and ox-CNF particles was the $\pi-\pi$ interactions, although one might also argue that hydrogen bonding between ox-CNF particles and UV stabilizers were likely to have formed. The extent of the $\pi-\pi$ interactions appeared to have been reduced in oxCNF/PMMA composites due to less perfect graphitic structure of oxCNF particles [11].

We also referred to fluorescence lifetime imaging microscopy (FLIM) in order to obtain further insight on the fluorescence lifetimes in PMMA, CNF/PMMA, and ox-CNF/PMMA composites. Note that FLIM experiments utilized very well focused laser and presented an average lifetime over the tested sample area, whereby time-resolved fluorescence experiments utilized a lamp which resulted in a more dispersed beam. A representative FLIM image for unfilled PMMA sample was presented in Fig. 6. The FLIM results revealed significantly similar values to those obtained in TRES experiments and the correlation coefficient between the lifetime values determined via FLIM and TRES experiments were determined to be around 0.991 .

Next, a few words are in order about the potential impact of the non-covalent interactions of UV stabilizers with PMMA chains and filler particles on photostability of the PMMA composites. It has been well documented that the presence of 'free' UV stabilizers is essential to obtain highly photostable polymers [27,28]. Hence, one may argue that above described interactions of UV stabilizers with CNF and ox-CNF particles, which reduce the amount of free UV stabilizers in PMMA matrix, could reduce the efficiency of the UV stabilizers which in turn would reduce the photostability of PMMA composites. On the other hand, recall that carbonaceous fillers in polymer matrices could also enhance the UV stability of polymers by absorbing a portion of incident UV radiation and dissipating as heat. Thus, the presence of carbonaceous fillers might create two simultaneous processes - reducing the efficiency of UV stabilizer molecules by interacting with them while absorbing a portion of the UV radiation - which were competing with each other. In view of these, it is usually very difficult to a priori estimate the impact of interactions between UV stabilizers and carbonaceous fillers on the photostability of polymers [29].

For the average emission lifetimes obtained by lifetime imaging, emission spectra in solution and solid-state and bright field and FLIM images for the PMMA and the composites please see the Supporting Information.

\section{Conclusions}

\subsection{The following conclusions can be drawn from this study}

1. FTIR analysis provided evidence for the formation of hydrogen bonding between the carbonyl groups of PMMA and carboxylic acid groups on ox-CNF. The presence of hydrogen bonding appeared to be instrumental in creating a better state of filler dispersion in ox-CNF/PMMA composites.

2. Fluorescence emission spectroscopy revealed that UV stabilizers interacted with PMMA chains via intermolecular hydrogen bonds and with CNF and ox-CNF filler particles via $\pi-\pi$ interactions.

\section{Acknowledgements}

OC would like to gratefully acknowledge the financial assistance from The Scientific \& Technological Research Council of Turkey (TUBITAK) in the form of a scholarship. SCJ acknowledges the CAREER program of National Science Foundation for providing partial financial assistance for this work.

\section{Appendix. Supplementary information}

Supplementary data related to this article can be found online at doi:10.1016/j.polymer.2011.09.011.

\section{References}

[1] Lee H-C, Akhtar MS, Park JG, Kim K-J, Lee SK, Yang O-B. J Nanosci Nanotechnol 2010;10:3502.

[2] Chen F, Wang B, Chen Y, Li L-J. Nano Lett 2007;7:3013.

[3] Gao J, Loi MA. Eur Phys J B 2010;75:121.

[4] Zeng J, Saltysiakb B, Johnson WS, Schiraldi DA, Kumar S. Composites Part B 2004;35:245.

[5] Das A, Hayvaci HT, Tiwari MK, Bayer IS, Erricolo D, Megaridis CM. J Colloid Interf Sci 2011;353:311.

[6] Liu J, Rasheed A, Minus ML, Kumar S. J Appl Polym Sci 2009;112:142.

[7] Sinha Ray S, Okamoto M. Prog Polym Sci 2003;28:1539.

[8] Gunes IS, Cao F, Jana SC. J Polym Sci Part B Polym Phys 2005;46:1437.

[9] Gunes IS, Cao F, Jana SC. Polymer 2008;49:2223.

[10] Jimenez GA, Jana SC. Compos Part A Appl Sci Manuf 2007;38:983.

[11] Jimenez GA, Jana SC. Carbon 2007;45:2079.

[12] Gunes IS, Pérez-Bolivar C, Cao F, Jimenez GA, Anzenbacher P, Jana SC. J Mater Chem 2010;20(3467).

[13] Morales-Teyssier O, Sánchez-Valdes S, Ramos-de Valle LF. Macromol Mater Eng 2006;291:1547.

[14] Gunes IS, Jimenez GA, Jana SC. Carbon 2009;47:981.

[15] Jung C, Jana SC, Gunes IS. Ind Eng Chem Res 2007;46:2413.

[16] Jimenez GA, Jana SC. Polym Eng Sci 2009;49:2020.

[17] Andrews RD. J Polym Sci Part C 1966;14:216.

[18] Jo WH, Cruz CA, Paul DR. J Polym Sci Part B Polym Phys 1989;27:1057.

[19] Coleman MM, Zarian J, Varnell DF, Painter PC. J Polym Sci Polym Lett Ed 1977; 15:745.

[20] Nyquist RA, Platt AE, Priddy DB. Appl Spectr 1982;36:417.

[21] Bart JCJ. Additives in polymers: Industrial analysis and applications. New York: Wiley; 2005. 785-786.

[22] Vogl O, Albertsson AC, Janovic Z. Polymer 1985;26:1288.

[23] Basf AG. Uvinul ${ }^{\circledR}$ light stabilizers technical information data sheet (EVP 004605e), http://www2.basf.us/additives/pdfs/uvinul_grades_4605e.pdf; 2005 (accessed on April 2, 2011).

[24] Ghiggino KP, Scully AD, Leaver IH. J Phys Chem 1986;90:5089.

[25] Woessner G, Goeller G, Rieker J, Hoier H, Stezowski JJ, Daltrozzo W, et al. J Phys Chem 1985;89:3629.

[26] Woessner G, Goeller G, Kellat P, Stezowski JJ, Hauser M, Klein UKA, et al. J Phys Chem 1984;88:5544.

[27] Heller HJ. Eur Polym J 1969;5(Suppl. 1):105.

[28] Heller HJ, Blattmann HR. Pure Appl Chem 1972;30:145.

[29] Allen NS, Edge M. Fundamentals of polymer degradation and stabilization. New York: Elsevier; 1992. 